Visión Antataura

Vol.4, No.2: 49-61

Diciembre 2020 - Mayo 2021

Panamá

ISSN 2520-9892

\title{
Familias de existencia y unicidad para el problema abstracto de Cauchy
}

\section{The existence and uniqueness of families for the abstract Cauchy problem}

\author{
Rodrigo Combe ${ }^{1}$ \\ ${ }^{1}$ Maestría en Matemática Pura y Maestría en Ingeniería Matemática; Profesor, Universidad de Panamá, Centro \\ Regional Universitario de Azuero, Departamento de Matemática; rodrigo.combe@up.ac.pa; \\ https://orcid.org/0000-0003-2004-4142
}

Resumen: El presente trabajo conlleva al estudio de las familias de existencia y unicidad de operadores lineales acotados definidos sobre espacios de Banach como una herramienta para manejar el problema abstracto de Cauchy. En este trabajo se presenta un sistema de operadores más general, la familia $E_{0}$ de existencia para un operador lineal $A$, de operadores lineales acotados de un espacio de Banach $Y$ (puede ser diferente de $X$ ) en $X$ como una nueva herramienta por tratar con Problema Abstracto de Cauchy. Esta idea es algo diferente de lo tradicional. También se estudia a su compañera familia de unicidad.

Palabras clave: problema abstracto de Cauchy, existencia, unicidad, operadores.

\begin{abstract}
The present work leads to the study of the families of existence and uniqueness of bounded linear operators defined on Banach spaces as a tool to handle the abstract Cauchy problem. In this work a more general system of operators is presented, the family $E_{0}$ of existence for a linear operator $A$, of bounded linear operators of a Banach space $Y$ (can be different from $X$ ) in $X$ as a new tool to deal with pProblem Cauchy abstract. This idea is somewhat different from the traditional one. Its companion family of uniqueness will be also studied.
\end{abstract}

Key words: abstract Cauchy problem, existence, uniqueness, operators.

\section{Introducción}

La teoría de ecuaciones diferenciales en espacios de Banach juega un papel importante en matemática. Un tema importante en esta teoría es el problema de abstracto de Cauchy. Durante las últimas décadas el estudio de algunas generalizaciones de los semigrupos fuertemente continuos clásicos; tales como los semigrupos integrados, los semigrupos regularizados y las familias de existencia de operadores lineales acotados en $X$, han llamado mucho la atención, porque estos nuevos tipos de familias de operadores equipan al Problema Abstracto de Cauchy con sistemas de operadores para controlar los 
problemas bien y mal propuestos. (DeLaubenfels, Sun y Wang, 1995; Cortés, Fraguela, Grebennikov, Morín y Oliveros, 2011).

Se estudia el Problema Abstracto de Cauchy (PAC)

$$
\left\{\begin{array}{l}
u^{\prime}(t)=A u(t), \quad t \geq 0 \\
u(0)=x
\end{array}\right.
$$

donde $A$ es un operador lineal en un espacio de Banach (Martínez, 2016). La finalidad es caracterizar las familias de existencia y unicidad para las soluciones de dicho problema.

\section{Familias de existencia y unicidad.}

A lo largo de este trabajo $X, Y$ son espacios de Banach, $L(Y, X)$ es el espacio de todos los operadores lineales acotados definidos de $Y$ en $X$ y $L(X, X)=L(X)$. Para un operador $A, D(A)$ denota el dominio de $A$ y $R(A)$ al rango de $A$. $[D(A)]$ define el espacio normado $D(A)$ con la norma gráfica

$$
\|x\|_{[D(A)]}=\|x\|+\|A x\|, \quad x \in D(A) .
$$

Cuando $C \in L(Y, X),[R(C)]$ denota el espacio de Banach $R(C)$ con la norma

$$
\|x\|_{[R(C)]}:=\inf \{\|x\| ; C y=x\} .
$$

Definición 2.1: Una solución de (PAC) es una función valuada en $X, u(t)$ continuamente diferenciable tal que $u(t) \in D(A)$ para todo $t \geq 0$ y tal que (PCA) se satisface.

Definición 2.2: Si para algún $a>0$, existe una aplicación $H:[0, \infty) \rightarrow L(Y, X)$ fuertemente continua de orden $O\left(e^{w t}\right)$ tal que para $\lambda>a$

$$
G(\lambda) y=\int_{0}^{\infty} e^{-\lambda t} H(t) y d t, \quad y \in Y
$$

con $G(\cdot):(a, \infty) \rightarrow L(Y, X)$, entonces se dice que $G \in L T_{W}-L(Y, X)$.

Definición 2.3: Sea $A$ como en (PAC). Sean $E_{0} \in L(Y, X)$ y $U_{0} \in L(X)$ inyectiva.

1. La familia fuertemente continua de operadores $\{E(t)\}_{t \geq 0} \subset L(Y, X)$ es llamada una familia $E_{0}$ de existencia para $A$, si para cada $y \in Y, t \geq 0$ 


$$
\int_{0}^{t} E(s) y d s \in D(A) \quad \text { y } \quad A\left(\int_{0}^{t} E(s) y d s\right)=E(t) y-E_{0} y .
$$

También se dice que $A$ tiene una familia $E_{0}$ de existencia $\{E(t)\}_{t \geq 0}$.

2. La familia fuertemente continua de operadores $\{U(t)\}_{t \geq 0} \subset L(X)$ es llamada una familia $U_{0}$ de unicidad para $A$, si para todo $x \in D(A)$ y para $t \geq 0$

$$
\int_{0}^{t} U(s) A x d s=U(t) x-U_{0} x .
$$

También se dice que $A$ tiene una familia $U_{0}$ de unicidad $\{U(t)\}_{t \geq 0}$.

$D_{E_{0}}(A)$ es el subespacio de $D(A)$ que consiste de aquellos elementos $x$ para los cuales $A x \in R\left(E_{0}\right)$.

\section{Teorema 2.4:}

1. Si existe una familia $E_{0}$ de existencia $\{E(t)\}_{t \geq 0}$ para $A$, entonces (PAC) admite una solución $u(\cdot)$ para todo $x \in D_{E_{0}}(A)$ que satisface:

$$
\|u(t)\|_{[D(A)]} \leq M(t)\left(\|A\|_{\left[R\left(E_{0}\right)\right]}+\|x\|\right), \quad t \geq 0
$$

para cierta función positiva localmente acotada $M(t)$ sobre $[0, \infty]$.

2. Si existe una familia $U_{0}$ de unicidad $\{U(t)\}_{t \geq 0}$ para $A$, entonces todas las soluciones de (PAC) son únicas.

\section{Demostración:}

1. Sea $x \in D_{E_{0}}(A)$. Sea $y_{0} \in Y$ tal que $A x=E_{0} y_{0}$. Para $t \geq 0$, se define

$$
u(t):=x+\int_{0}^{t} E(s) y_{0} d s .
$$

Al derivar la ecuación ( $E c$ 2.4) se obtiene que $u^{\prime}(t)=E(t) y_{0}$. Además,

$$
\begin{aligned}
A u(t) & =A\left(x+\int_{0}^{t} E(s) y_{0} d s\right)=A x+A\left(\int_{0}^{t} E(s) y_{0} d s\right) \\
& =E_{0} y_{0}+E(t) y_{0}-E_{0} y_{0}=E(t) y_{0} .
\end{aligned}
$$

Por lo tanto, $u$ definida por la ecuación (Ec 2.4) satisface (PAC). Obsérvese que $\|E(t)\|$ es localmente acotada sobre $[0, \infty]$, debido al Teorema de Banach - Steinhaus (Kreyszig, 1989). Así pues, la expresión (Ec 2.3) se sigue de la arbitrariedad de $y_{0}$. 
2. Sea $u$ una solución de (PAC) con $x=0$. Si se despeja $U(t) x$ de la ecuación (Ec 2.2) y se cambia $t$ por $t-s$, se obtiene

$$
U(t-s) x=U_{0} x+\int_{0}^{t-s} U(s) A x d s
$$

y derivando con respecto a la variable $s$

$$
\frac{d}{d s} U(t-s) x=-U(t-s) A x .
$$

Por lo tanto,

$$
\frac{d}{d s} U(t-s)=-U(t-s) A .
$$

De este último resultado, se deduce que

$$
\frac{d}{d s}[U(t-s) u(s)]=-U(t-s) A u(s)+U(t-s) u^{\prime}(s)=0, \quad t \geq s \geq 0 .
$$

Por consiguiente, $U_{0} u(t)=U(t) u(0)=0$ para $t \geq 0$. La inyectividad de $U_{0}$ nos asegura que $u(t) \equiv 0$.

El siguiente ejemplo muestra que la elección de un espacio de Banach diferente de $X$ produce un conjunto grande de datos iniciales.

Ejemplo 2.5: Sea $A$ un operador lineal en $C_{0}(\mathbb{R})$, definido por $(A f)(x):=x f(x), x \in \mathbb{R}$ para toda

$$
f \in D(A):=\left\{f \in C_{0}(\mathbb{R}) ; x f(x) \in C_{0}(\mathbb{R})\right\} .
$$

No es difícil verificar que $A$ tiene una familia $E_{0}$ de existencia $\{E(t)\}_{t \geq 0}$ de operadores en $L\left(C_{b}(\mathbb{R}), C_{0}(\mathbb{R})\right)$ y una familia $U_{0}$ de unicidad $\{U(t)\}_{t \geq 0}$ de operadores en $L\left(C_{0}(\mathbb{R})\right)$. Para $x \in \mathbb{R}$ y $t \geq 0$ se tiene que

$$
\begin{gathered}
(E(t) f)(x):=e^{-x^{2}} e^{t x} f(x), \quad f \in C_{b}(\mathbb{R}) \\
U(t):=\left.E(t)\right|_{C_{0}(\mathbb{R})} \\
E_{0}=E(0), \quad U_{0}=U(0) .
\end{gathered}
$$

Por el Teorema 2.4, se deduce que (PAC) admite una única solución siempre que el valor inicial esté en

$$
D_{E_{0}}(A)=\left\{f \in C_{0}(\mathbb{R}) ; x e^{x^{2}} f(x) \text { es acotado en } \mathbb{R}\right\}
$$


Ciertamente, $\{U(t)\}_{t \geq 0}$ es también una familia $U_{0}$ de existencia en $L\left(C_{0}(\mathbb{R})\right)$ para $A$. En este caso, se tiene que

$$
D_{E_{0}}(A)=U_{0}(D(A))=\left\{f \in C_{0}(\mathbb{R}) ; x e^{x^{2}} f(x) \in C_{0}(\mathbb{R})\right\} .
$$

\section{Caracterizaciones usando las Transformadas de Laplace.}

Lema 3.1: Sean $k$ una constante y $h_{1}, h_{2} \in C([0, \infty], X)$ con $\left\|h_{1}(t)\right\|,\left\|h_{2}(t)\right\| \leq k e^{w t}$ para $t \geq 0$ y algún $w>0$. Sea $A$ un operador cerrado en $X$ tal que para todo $\lambda>w$,

$$
\int_{0}^{\infty} e^{-\lambda t} h_{1}(t) d t \in D(A) \quad \text { y } \quad A \int_{0}^{\infty} e^{-\lambda t} h_{1}(t) d t=\int_{0}^{\infty} e^{-\lambda t} h_{2}(t) d t .
$$

Entonces para todo $t \geq 0, h_{1}(t) \in D(A)$ y $A h_{1}(t)=h_{2}(t)$ (Xiao and Liang, 1998).

Demostración: Es una consecuencia directa del Teorema de Unicidad de Transformadas de Laplace y del hecho de que el operador $A$ es cerrado (Peng y Chung, 1998).

A continuación, se caracterizarán las familias $E_{0}$ de existencia $O\left(e^{w t}\right)$ en lenguaje de transformadas de Laplace.

Teorema 3.2: Sean $w \in \mathbb{R}$ y sea $\{E(t)\}_{t \geq 0}$ una familia de operadores fuertemente continuos en $L(Y, X)$ tal que $\|E(t)\| \leq k e^{w t}$, para una constante $k$ y $t \geq 0$. Supongamos que $A$ es cerrado, y $\lambda-A$ es inyectiva para $\lambda>a$ (para algún $a>\max (w, 0))$. Entonces las siguientes condiciones son equivalentes:

a. $\{E(t)\}_{t \geq 0}$ es una familia $E_{0}$ de existencia para $A$.

b. $R\left(E_{0}\right) \subset R(\lambda-A)$ para $\lambda>a$ y

$$
(\lambda-A)^{-1} E_{0} y=\int_{0}^{\infty} e^{-\lambda t} E(t) y d t, \quad y \in Y .
$$

Demostración: Primeramente, supongamos que $\{E(t)\}_{t \geq 0}$ es una familia $E_{0}$ de existencia para $A$ y por lo tanto, se verifica la ecuación (Ec 2.1):

$$
A\left(\int_{0}^{t} E(s) y d s\right)=E(t) y-E_{0} y .
$$

Multiplicando por $e^{-\lambda t}$ y despejando $E_{0} y$ se obtiene 
54. Visión Antataura, Vol.4, No.2, Diciembre 2020 - Mayo 2021

$$
e^{-\lambda t} E_{0} y=e^{-\lambda t} E(t) y-e^{-\lambda t} A\left(\int_{0}^{t} E(s) y d s\right),
$$

pero como $A$ es lineal

$$
e^{-\lambda t} E_{0} y=e^{-\lambda t} E(t) y-A\left(e^{-\lambda t} \int_{0}^{t} E(s) y d s\right)
$$

Para $\lambda>a$,

$$
\begin{aligned}
\int_{0}^{\infty} e^{-\lambda t} E_{0} y d t & =\int_{0}^{\infty} e^{-\lambda t} E(t) y d t-A\left(\int_{0}^{\infty} e^{-\lambda t} \int_{0}^{t} E(s) y d s d t\right) \\
E_{0} y \int_{0}^{\infty} e^{-\lambda t} d t & =\int_{0}^{\infty} e^{-\lambda t} E(t) y d t-A\left(\int_{0}^{\infty} e^{-\lambda t} \int_{0}^{t} E(s) y d s d t\right) \\
\frac{E_{0} y}{\lambda} & =\int_{0}^{\infty} e^{-\lambda t} E(t) y d t-A\left(\int_{0}^{\infty} e^{-\lambda t} \int_{0}^{t} E(s) y d s d t\right) .
\end{aligned}
$$

Usando integración por partes en la integral

$$
\int_{0}^{\infty} e^{-\lambda t} \int_{0}^{t} E(s) y d s d t
$$

con

$$
\begin{array}{rlrl}
u & =\int_{0}^{t} E(s) y d s & d v & =e^{-\lambda t} d t \\
d u & =E(t) y d t & v & =-\frac{e^{-\lambda t}}{\lambda}
\end{array}
$$

se obtiene

$$
\int_{0}^{\infty} e^{-\lambda t} \int_{0}^{t} E(s) y d s d t=-\left.\frac{e^{-\lambda t}}{\lambda} \int_{0}^{t} E(s) y d s\right|_{0} ^{\infty}+\int_{0}^{\infty} \frac{e^{-\lambda t}}{\lambda} E(t) y d t
$$

La hipótesis $\|E(t)\| \leq k e^{w t}$, para $t \geq 0$, nos indica que

$$
\begin{aligned}
\left\|-\frac{e^{-\lambda t}}{\lambda} \int_{0}^{t} E(s) y d s\right\| & \leq-\frac{e^{-\lambda t}}{\lambda}\left(\int_{0}^{t}\|E(s)\| d s\right)\|y\| \\
& \leq-\frac{e^{-\lambda t}}{\lambda} k\|y\| \int_{0}^{t} e^{w t} d s \\
& \leq-\frac{k\|y\| e^{-\lambda t}\left(e^{w s}-1\right)}{\lambda w} \rightarrow 0
\end{aligned}
$$

de donde resulta que 
55. Visión Antataura, Vol.4, No.2, Diciembre 2020 - Mayo 2021

$$
-\left.\frac{e^{-\lambda t}}{\lambda} \int_{0}^{t} E(s) y d s\right|_{0} ^{\infty} \rightarrow 0
$$

Por lo tanto,

$$
\int_{0}^{\infty} e^{-\lambda t} \int_{0}^{t} E(s) y d s d t=\frac{1}{\lambda} \int_{0}^{\infty} e^{-\lambda t} E(t) y d t .
$$

De las ecuaciones ( $E c$ 2.5) y (Ec 2.6) se observa que

$$
\frac{E_{0} y}{\lambda}=\int_{0}^{\infty} e^{-\lambda t} E(t) y d t-A\left(\frac{1}{\lambda} \int_{0}^{\infty} e^{-\lambda t} E(t) y d t\right) .
$$

Como $A$ es un operador lineal,

$$
\begin{aligned}
E_{0} y & =\lambda \int_{0}^{\infty} e^{-\lambda t} E(t) y d t-A\left(\int_{0}^{\infty} e^{-\lambda t} E(t) y d t\right) \\
& =(\lambda-A) \int_{0}^{\infty} e^{-\lambda t} E(t) y d t
\end{aligned}
$$

de donde se deduce que

$$
(\lambda-A)^{-1} E_{0} y=\int_{0}^{\infty} e^{-\lambda t} E(t) y d t
$$

Recíprocamente, supongamos que $R\left(E_{0}\right) \subset R(\lambda-A)$ para $\lambda>a \mathrm{y}$

$$
(\lambda-A)^{-1} E_{0} y=\int_{0}^{\infty} e^{-\lambda t} E(t) y d t, \quad y \in Y .
$$

De la hipótesis y de la ecuación (Ec 2.6) se observa que

$$
\lambda^{-1}(\lambda-A)^{-1} E_{0} y=\int_{0}^{\infty} e^{-\lambda t} \int_{0}^{t} E(s) y d s d t
$$

y multiplicando por $A$ se obtiene que

$$
\lambda^{-1} A(\lambda-A)^{-1} E_{0} y=A \int_{0}^{\infty} e^{-\lambda t} \int_{0}^{t} E(s) y d s d t . \quad \text { (Ec 2.7) }
$$

Además,

$$
\begin{aligned}
-\lambda^{-1} E_{0} y+(\lambda-A)^{-1} E_{0} y & =\frac{-\lambda E_{0} y+A E_{0} y+\lambda E_{0} y}{\lambda(\lambda-A)} \\
& =\frac{A E_{0} y}{\lambda(\lambda-A)} \\
& =\lambda^{-1} A(\lambda-A)^{-1} E_{0} y
\end{aligned}
$$

y como 
56. Visión Antataura, Vol.4, No.2, Diciembre 2020 - Mayo 2021

$$
\lambda^{-1} E_{0} y=\int_{0}^{\infty} e^{-\lambda t} E_{0} y d t \quad y \quad(\lambda-A)^{-1} E_{0} y=\int_{0}^{\infty} e^{-\lambda t} E(t) y d t,
$$

se tiene que

$$
\begin{aligned}
\lambda^{-1} A(\lambda-A)^{-1} E_{0} y & =-\int_{0}^{\infty} e^{-\lambda t} E_{0} y d t+\int_{0}^{\infty} e^{-\lambda t} E(t) y d t \\
& =\int_{0}^{\infty} e^{-\lambda t}\left[E(t) y-E_{0} y\right] d t
\end{aligned}
$$

(Driver, 2003). De las ecuaciones (Ec 2.7) y (Ec 2.8) se obtiene que

$$
A \int_{0}^{\infty} e^{-\lambda t} \int_{0}^{t} E(s) y d s d t=\int_{0}^{\infty} e^{-\lambda t}\left[E(t) y-E_{0} y\right] d t
$$

y se deduce en virtud del Lema 3.1, que para todo $t \geq 0, y \in Y$,

$$
\int_{0}^{t} E(s) y d s \in D(A)
$$

y la ecuación (Ec 2.1) se verifica.

Teorema 3.3: Si (a) o (b) del Teorema 3.2 se verifican, entonces para cada $x \in D_{E_{0}}(A)$, (PAC) admite una única solución exponencialmente acotada $u(t)$ tal que

$$
\|u(t)\|_{D(A)} \leq k M(t)\left(\|x\|+\|A\|_{\left[R\left(E_{0}\right)\right]}\right), \quad t \geq 0
$$

donde $k$ es una constante y

$$
M(t):=\left\{\begin{array}{cl}
e^{\max (w, 0) t} & \text { si } w \neq 0 \\
t & \text { si } w=0
\end{array}\right.
$$

Si $x \in R\left(E_{0}\right)$, entonces

$$
\|u(t)\| \leq k e^{w t}\|x\|_{\left[R\left(E_{0}\right)\right]}, \quad t \geq 0 .
$$

Demostración: Del enunciado y demostración del Teorema 2.4, se obtienen los resultados requeridos, excepto la desigualdad $(E c 2.9)$. Sea $\lambda>\max (w, 0)$. Al multiplicar la ecuación (Ec 2.4) por $e^{-\lambda t}$ e integrando se tiene que

$$
\begin{aligned}
\int_{0}^{\infty} e^{-\lambda t} u(t) d t & =\int_{0}^{\infty} e^{-\lambda t} x d t+\int_{0}^{\infty} e^{-\lambda t} \int_{0}^{t} E(s) y_{0} d s d t \\
& =\lambda^{-1} x+\int_{0}^{\infty} e^{-\lambda t} \int_{0}^{t} E(s) y_{0} d s d t
\end{aligned}
$$

Calculando la segunda integral mediante integración por partes, se verifica que 


$$
\int_{0}^{\infty} e^{-\lambda t} u(t) d t=\lambda^{-1} x+\lambda^{-1} \int_{0}^{\infty} e^{-\lambda t} E(t) y_{0} d t
$$

Pero del Teorema 3.2, se sabe que

$$
(\lambda-A)^{-1} E_{0} y=\int_{0}^{\infty} e^{-\lambda t} E(t) y d t, \quad y \in Y, \lambda>a
$$

y por lo tanto

$$
\int_{0}^{\infty} e^{-\lambda t} u(t) d t=\lambda^{-1} x+\lambda^{-1}(\lambda-A)^{-1} E_{0} y_{0} .
$$

Obsérvese que $E_{0} y_{0}=A x$ y así

$$
\begin{aligned}
\int_{0}^{\infty} e^{-\lambda t} u(t) d t & =\lambda^{-1} x+\lambda^{-1}(\lambda-A)^{-1} A x \\
& =\frac{\lambda x-A x+A x}{\lambda(\lambda-A)} \\
& =(\lambda-A)^{-1} A .
\end{aligned}
$$

Cuando $x \in R\left(E_{0}\right)$, si se toma un $y_{1} \in Y$ tal que $E_{0} y_{1}=x$, entonces por el Teorema 3.2 para $\lambda>a$,

$$
\begin{aligned}
(\lambda-A)^{-1} x & =(\lambda-A)^{-1} E_{0} y_{1} \\
& =\int_{0}^{\infty} e^{-\lambda t} E(t) y_{1} d t .
\end{aligned}
$$

De la ecuación (Ec 2.10) y el Teorema de Unicidad para Transformadas de Laplace, se tiene que $u(t) \equiv E(t) y_{1}$. Por consiguiente, la ecuación (Ec 2.6) se obtiene de la arbitrariedad de $y_{1}$.

El próximo teorema es una caracterización de una familia $U_{0}$ de unicidad $O\left(e^{w t}\right)$ para $A$ en términos de transformadas de Laplace.

Teorema 3.4: Sea $\{U(t)\}_{t \geq 0}$ una familia fuertemente continua de operadores en $L(X)$ tal que $\|U(t)\| \leq k e^{w t}$, para una constante $k$, para $t \geq 0$ y algún $w \geq 0$. Entonces, los siguientes enunciados son equivalentes:

a. $\{U(t)\}_{t \geq 0}$ es una familia $U_{0}$ de unicidad para $A$. 
b. Para $\lambda>w, \quad U_{0} x=\int_{0}^{\infty} e^{-\lambda t} U(t)(\lambda-A) x d t$ para $x \in D(A)$.

Demostración: Sea $\{U(t)\}_{t \geq 0}$ es una familia $U_{0}$ de unicidad para $A$. Luego se verifica la ecuación (Ec 2.2). Si se multiplica la ecuación (Ec 2.2) por $e^{-\lambda t}$ se obtiene que

$$
e^{-\lambda t} \int_{0}^{t} U(s) A x d s=e^{-\lambda t} U(t) x-e^{-\lambda t} U_{0} x
$$

Integrando esta expresión para $\lambda>0$ se tiene que

$$
\begin{aligned}
\int_{0}^{\infty} e^{-\lambda t} \int_{0}^{t} U(s) A x d s d t & =\int_{0}^{\infty} e^{-\lambda t} U(t) x d t-\int_{0}^{\infty} e^{-\lambda t} U_{0} x d t \\
& =\int_{0}^{\infty} e^{-\lambda t} U(t) x d t-U_{0} x \int_{0}^{\infty} e^{-\lambda t} d t \\
\int_{0}^{\infty} e^{-\lambda t} \int_{0}^{t} U(s) A x d s d t & =\int_{0}^{\infty} e^{-\lambda t} U(t) x d t-\frac{U_{0} x}{\lambda}
\end{aligned}
$$

Al despejar $U_{0} x$ se obtiene que

$$
U_{0} x=\int_{0}^{\infty} e^{-\lambda t} U(t) \lambda x d t-\int_{0}^{\infty} \lambda e^{-\lambda t} \int_{0}^{t} U(s) A x d s d t
$$

Calculando

$$
\int_{0}^{\infty} \lambda e^{-\lambda t} \int_{0}^{t} U(s) A x d s d t
$$

mediante integración por partes con

$$
\begin{array}{rlrl}
u & =\int_{0}^{t} U(s) A x d s & d v & =\lambda e^{-\lambda t} d t \\
d u & =U(t) A x d t & v & =-e^{-\lambda t}
\end{array}
$$

se deduce que

$$
\int_{0}^{\infty} \lambda e^{-\lambda t} \int_{0}^{t} U(s) A x d s d t=-\left.e^{-\lambda t} \int_{0}^{t} U(s) A x d s\right|_{0} ^{\infty}+\int_{0}^{\infty} e^{-\lambda t} U(t) A x d t .
$$

Debido a $\|U(t)\| \leq k e^{w t}$, para una constante $k$, para $t \geq 0$ y algún $w \geq 0$ se tiene que

$$
\begin{aligned}
\left\|-e^{-\lambda t} \int_{0}^{t} U(s) A x d s\right\| & \leq-e^{-\lambda t}\|A x\| \int_{0}^{t}\|U(s)\| d s \\
& \leq-k e^{-\lambda t}\|A x\| \int_{0}^{t} e^{w s} d s
\end{aligned}
$$


59. Visión Antataura, Vol.4, No.2, Diciembre 2020 - Mayo 2021

$$
\leq-\frac{k e^{-\lambda t}\|A x\|\left(e^{w t}-1\right)}{w} \rightarrow 0
$$

por lo que

$$
-\left.e^{-\lambda t} \int_{0}^{t} U(s) A x d s\right|_{0} ^{\infty} \rightarrow 0
$$

y así

$$
\int_{0}^{\infty} \lambda e^{-\lambda t} \int_{0}^{t} U(s) A x d s d t=\int_{0}^{\infty} e^{-\lambda t} U(t) A x d t .
$$

De las ecuaciones (Ec 2.11) y (Ec 2.12) se desprende que

$$
\begin{aligned}
& U_{0} x=\int_{0}^{\infty} e^{-\lambda t} U(t) \lambda x d t-\int_{0}^{\infty} e^{-\lambda t} U(t) A x d t \\
& U_{0} x=\int_{0}^{\infty} e^{-\lambda t} U(t)(\lambda-A) x d t .
\end{aligned}
$$

Recíprocamente, supongamos que para $x \in D(A)$ y para $\lambda>w$, se tiene que:

$$
U_{0} x=\int_{0}^{\infty} e^{-\lambda t} U(t)(\lambda-A) x d t
$$

Por otro lado,

$$
\begin{aligned}
\lambda \int_{0}^{\infty} e^{-\lambda t}\left[U(t) x-U_{0} x\right] d t & =\lambda \int_{0}^{\infty} e^{-\lambda t} U(t) x d t+\lambda \int_{0}^{\infty} e^{-\lambda t} U_{0} x d t \\
& =\lambda \int_{0}^{\infty} e^{-\lambda t} U(t) x d t+U_{0} x \\
& =\lambda \int_{0}^{\infty} e^{-\lambda t} U(t) x d t+\int_{0}^{\infty} e^{-\lambda t} U(t)(\lambda-A) x d t \\
& =\int_{0}^{\infty} e^{-\lambda t} U(t) A x d t . \quad \text { (Ec 2.13) }
\end{aligned}
$$

De las ecuaciones ( $E c$ 2.12) y (Ec 2.13) se infiere que

$$
\int_{0}^{\infty} \lambda e^{-\lambda t} \int_{0}^{t} U(s) A x d s d t=\lambda \int_{0}^{\infty} e^{-\lambda t}\left[U(t) x-U_{0} x\right] d t
$$

y por el Teorema de Unicidad para Transformadas de Laplace se verifica que $\{U(t)\}_{t \geq 0}$ es una familia $U_{0}$ de unicidad para $A$. 
Observación: Si $X=Y$, entonces $E_{0}$ es inyectiva y $E_{0} A \subset A E_{0}$. Además, es evidente que $\{E(t)\}_{t \geq 0}$ en el Teorema 2.4 es también una familia $E_{0}$ de unicidad exponencialmente acotada para $A$.

Ejemplo 3.5: Sea $X=\ell^{2}$ el conjunto de todas las sucesiones $\left\{u_{m}\right\}_{m \geq 1}$ de números complejos con

$$
\left\|\left\{u_{m}\right\}_{m \geq 1}\right\|:=\sum_{m=1}^{\infty}\left|u_{m}\right|^{2}<\infty .
$$

Considere los operadores $A$ y $E_{0}$ definidos por

$$
A\left\{u_{m}\right\}=\left\{\widetilde{u_{m}}\right\}, \quad E_{0}\left\{u_{m}\right\}=\left\{\overline{u_{m}}\right\} \text { para todo }\left\{u_{m}\right\} \in X,
$$

donde $\widetilde{u_{1}}=u_{1}, \overline{u_{1}}=0 \quad$ y

$$
\widetilde{u_{m}}=\overline{u_{m}}=\left\{\begin{array}{cl}
0 & \text { si } m \text { es par } \\
u_{i+1} & \text { si } m \text { es impar. }
\end{array}\right.
$$

Aplicando estos operadores a una sucesión cualquiera $\left\{u_{m}\right\} \in X$ se tiene que

$$
A\left\{u_{m}\right\}=\left\{\widetilde{u_{m}}\right\}=\left(u_{1}, 0, u_{2}, 0, u_{3}, 0, u_{4}, 0, \ldots\right)
$$

y

$$
E_{0}\left\{u_{m}\right\}=\left\{\overline{u_{m}}\right\}=\left(0,0, u_{2}, 0, u_{3}, 0, u_{4}, 0, \ldots\right)
$$

No es difícil verificar que $A, E_{0} \in L(X)$ es inyectiva, pero no sobreyectiva. Además

$$
\begin{gathered}
E_{0} A=A E_{0}=\left(0,0,0,0, u_{2}, 0,0, u_{3}, 0,0, u_{4}, 0,0, \ldots\right), \\
D_{E_{0}}(A)=\left\{\left\{v_{m}\right\} \in X: v_{1}=0\right\}
\end{gathered}
$$

y

$$
E_{0} D(A)=\left\{\left\{v_{m}\right\} \in X: v_{1}=v_{m}=0 \text { para cualquier } m \text { par }\right\} .
$$

Por lo tanto, $D_{E_{0}}(A) \neq E_{0} D(A)$.

\section{Referencias bibliográficas}

Cortés, M., Fraguela, A., Grebennikov, A., Morín, M. y Oliveros, J. (2011). Solución estable del problema de Cauchy para la ecuación de Laplace usando potenciales de superficie. Lecturas Matemáticas, 32, 61-77.

DeLaubenfels, R., Sun, G. y Wang, S. (1995). Regularized semigroups, existence families and the abstract Cauchy problem. Differential and Integral Equations, 8 (6), 1477-1496. 
Driver, B. (2003). Analysis tools with applications. New York: Springer's Internal Project.

Kreyszig, E. (1989). Introductory functional analysis with applications. New York: John Wiley y Sons.

Martínez, M. (2016). On Asymptotic behavior of one-parameter families of bounded operators on Banach spaces. Tesis Doctoral. Universidad de Zaragoza.

Peng, J., Chung, S. K. (1998). Laplace transforms and generators of semigroups of operators. Proceedings of the American Mathematical Society. 126 (8), 2407-2416.

Xiao, T. J., Liang, J. (1998). Differential operators and C-Wellposedness of complete second order abstract Cauchy problems. Pacific Journal of Mathematics. 186 (1), 167-200. 